\section{Inhibition of fibrous adhesion formation in the temporo- mandibular joint of tenascin-C knockout mice}

\author{
Y. Shinohara, K. Okamoto, Y. Goh, \\ N. Kiga, I. Tojyo, S. Fujita \\ Department of Oral Maxillofacial Surgery, \\ Wakayama Medical University, Japan
}

\begin{abstract}
Tenascin-C (TNC) is a large hexameric extracellular matrix glycoprotein that is expressed in developing organs and tumors. It has been reported that TNC is expressed in inflamed synovial membranes and deformed discs of temporomandibular joint (TMJ) disorder. However, the role of TNC in TMJ is not fully known. In this study, the role of TNC in fibrous adhesion formation of TMJ was examined using TNC knockout (TNCKO) mice. Hypermobility was produced by excessive mouth opening method on the TMJ of both wild-type (WT) and TNCKO mice. TMJ wound healing was compared histologically, and the expression of TNC, fibronectin (FN), and $\alpha$ smooth muscle actin ( $\alpha$-SMA) in the wounded TMJ was examined by immunohistochemical and immunoblot analyses. Based on histologic analysis, fibrous adhesions were observed in the TMJ of both TNCKO and wild-type (WT) mice after excessive mouth opening. However, fibrous adhesion formation in TNCKO mice occurred later than in WT mice. TNC was expressed in the wounded TMJ disc and mandibular fossa. Although FN and $\alpha$-SMA expression in the TMJ of TNCKO and WT mice was up-regulated after excessive mouth opening, FN and $\alpha$-SMA protein levels were higher in WT mice at the same time points. In the wounded TMJ, TNC appears to enhance the expression of FN and $\alpha$-SMA, and a lack of TNC may reduce fibrous adhesion formation in the TMJ. TNC plays an important role in TMJ wound healing, especially for wounds generated by mechanical stress.
\end{abstract}

\section{Introduction}

A broad spectrum of temporomandibular joint (TMJ) disorders (TMD) is recognized, ranging from an occasional painless click to degenerative diseases with condylar and mandibular fossa resorption. Adhesion formation is a degenerative change that plays a role in pain, as well as in the progression of disc pathology by mechanical limitation of disc function. ${ }^{1-3}$ Remodeling is an essential biological response to normal functional demands, homeostasis of joint structure, and function and occlusal relationships. ${ }^{4}$ Arnett et al. proposed an explanation for the pathophysiology of degenerative changes as one that results from dysfunctional articular remodeling due to excessive or sustained physical stress to the TMJ articular structures that exceeds the normal adaptive capacity. ${ }^{5}$ Increased loading in the TMJ may stimulate remodeling, involving increased synthesis of extracellular matrices. ${ }^{6}$ Microtrauma produced during mandibular movement, synovitis, or a masticatory muscle disorder may occasionally lead to unfavorable biomechanics of the TMJ complex, leading to excessive mechanical stress and resulting in adhesion formation. Adhesion formation is a process that can occur during wound healing, and is characterized by microtrauma-induced elevation of extracellular matrix synthesis.

Tenascin-C (TNC) is a disulfide-bonded hexameric matrix component composed of subunits with molecular weights in the range of $120-300 \mathrm{kDa} .^{7,8} \mathrm{TNC}$ is highly expressed during embryonic development, tissue repair, and in pathological conditions such as chronic inflammation. TNC interacts with several other extracellular matrix molecules and cellsurface receptors, thus affecting tissue architecture, tissue resilience, and cell responses. ${ }^{9}$ In TMJ tissues, immunolabeled TNC is present at low levels in normal healthy synovial membranes and discs, while it is up-regulated in the inflamed synovial membranes and deformed discs of TMD. ${ }^{10}$ TNC regulates cell differentiation, division, migration, binding, and regulatory processes during the formation of bone matrices in the mandibular condyle. ${ }^{11}$ Although these previous studies strongly suggested that TNC is involved in TMD, it has not yet been determined whether TNC promotes fibrous adhesion formation in the TMJ. Fibronectin (FN) is a large, adhesive glycoprotein that is found in the extracellular matrix of a variety of tissues, including articular tissues of the TMJ. ${ }^{12}$ Normal TMJ tissue weakly expresses FN, with more pronounced expression in TMD tissue. ${ }^{13}$ FN is composed of heterodimers connected by 2 disulfide bonds near their carboxyl ends. FN is a polyvalent molecule composed of several domains that contain binding sites for a large number of extracellular matrix components, such as collagen, heparin, and fibrin. FN molecules also contain domains that facilitate cell adhesion via cell surface receptors, called integrins. ${ }^{14}$ In an in vitro study, Dijkgraaf et al. demonstrated that FN may be involved in adhesion formation via free radical-mediated crosslinking. ${ }^{15}$ They observed that during elevated oxidative stress, fragments of FN form a high molecular weight
Correspondence: Dr. Yuji Shinohara, Department of Oral Maxillofacial Surgery, Wakayama Medical University, 811-1 Kimiidera, Wakayama 6418509, Japan.

Tel./Fax: +81.73.441.0643.

E-mail: yujii@wakayama-med.ac.jp

Key words: Temporomandibular joint, excessive mouth opening, fibrous adhesion, tenascin-C, fibronectin, $\alpha$-smooth muscle actin.

Acknowledgments: We are indebted to Prof. S. Saika and Y. Okada, Department of Ophthalmology, Wakayama Medical University for providing the TNCKO mice.

This work was partly supported by KAKENHI from the Ministry of Education, Culture, Sports, Science and Technology, and the Japanese Government.

Contributions: YS, SF, study design; YS, KO, YG, NK, IT, experimental procedures, immunohistochemical processing, microscopic analysis, data collection and/or preparation of figures; YS contributed especially to western blot analysis. All authors have reviewed and contributed to the different draft versions of the manuscript and have read and approved the final manuscript.

Conflicts of interest: the authors declare no conflicts of interests.

Received for publication: 29 October 2013. Accepted for publication: 9 September 2014.

This work is licensed under a Creative Commons Attribution NonCommercial 3.0 License (CC BYNC 3.0).

(C) Copyright Y. Shinohara et al., 2014

Licensee PAGEPress, Italy

European Journal of Histochemistry 2014; 58:2337 doi:10.4081/ejh.2014.2337

aggregate. Therefore, the authors argued that these crosslinked proteins might serve as the initial scaffolding for the development of adhesions frequently seen in TMD.

The term myofibroblast was proposed for fibroblastic cells within granulation tissue that exhibited cytoplasmic microfilamentous apparatus. ${ }^{16-18}$ It was later discovered that the microfilament structure of myofibriblasts was $\alpha$ smooth muscle actin ( $\alpha$-SMA), the actin isoform typical of smooth muscle cells in vessel walls that serves as a contractile smooth muscle protein; $\alpha$-SMA is currently accepted the most reliable marker of myofibroblasts. Myofibroblasts have been seen in non-musculoskeletal pathologic fibrotic conditions characterized by contractures such as liver cirrhosis, pulmonary fibrosis, and corneal fibrosis. ${ }^{19-21}$

Several experimental animal models involving the use a mandibular displacement appliance, ${ }^{22}$ alterations to the occlusion, ${ }^{23}$ or tooth 
extraction $^{24}$ have implicated the involvement of mechanical loading to the articular surface in histological changes of the TMJ, including the synovial membrane and the articular cartilage. Recently, a forced-jaw-opening protocol has been shown to be effective in inducing articular fibrous adhesions in the TMJ. ${ }^{25,26}$ These two studies are important since they clearly demonstrate that adverse joint loading without any surgical manipulation of the joint tissues can induce fibrous adhesion in vivo. TNC knockout (TNCKO) mice develop normally, ${ }^{27}$ but exhibit abnormal behavior, defects in the structure and repair of neuromuscular junctions, ${ }^{28}$ and defective recovery from snake venom-induced glomerulonephritis ${ }^{29}$ and chemically induced dermatitis. ${ }^{30}$ Moreover, it was reported that the absence of TNC suppresses the development of fibrosis in mice. ${ }^{31}$ The definite role of TNC in fibrous adhesions of the TMJ is not yet known. To elucidate this, we compared the wound healing process of the TMJ in TNCKO mice ${ }^{32}$ and congenic wild-type (WT) mice after excessive mouth opening. Furthermore, we investigated the expression of TNC, FN and $\alpha$-SMA, which are important extracellular matrix proteins, in wound healing.

\section{Materials and Methods}

\section{Animals and experimental protocol}

Male TNCKO mice of the C57BL/6 strain and WT littermates ( 8 weeks of age) were used and maintained according to guidelines approved by the DNA Recombination Experiment Committee and the Animal Care and Use Committee of Wakayama Medical University. Excessive mouth opening was induced by opening the mouth widely $(10 \mathrm{~mm})$ under sodium pentobarbital anesthesia. This procedure was repeated 40 times, once daily for 20 consecutive days. Untreated (control) and treated mice were sacrificed after $1,7,12$, or 20 days of the excessive mouth opening procedure. A total of 30 mice ( 15 TNCK0 and 15 WT) were examined histologically, and additional 20 mice (10 TNCKO and 10 WT) were used for quantitative analyses.

\section{Histochemical analysis}

To compare histological changes as a result of TMJ loading by excessive mouth opening, histological analysis was performed. All mice were perfused via the left ventricle with $4 \%$ paraformaldehyde (PFA) under sodium pentobarbital anesthesia. TMJ samples were dissected out and fixed in a solution of 4\% PFA for $24 \mathrm{~h}$, and then washed for $24 \mathrm{~h}$ in phosphatebuffered saline (PBS, pH 7.4). Samples were decalcified in a $4 \%$ EDTA solution at $4^{\circ} \mathrm{C}$ for 4 weeks. The samples were dehydrated and
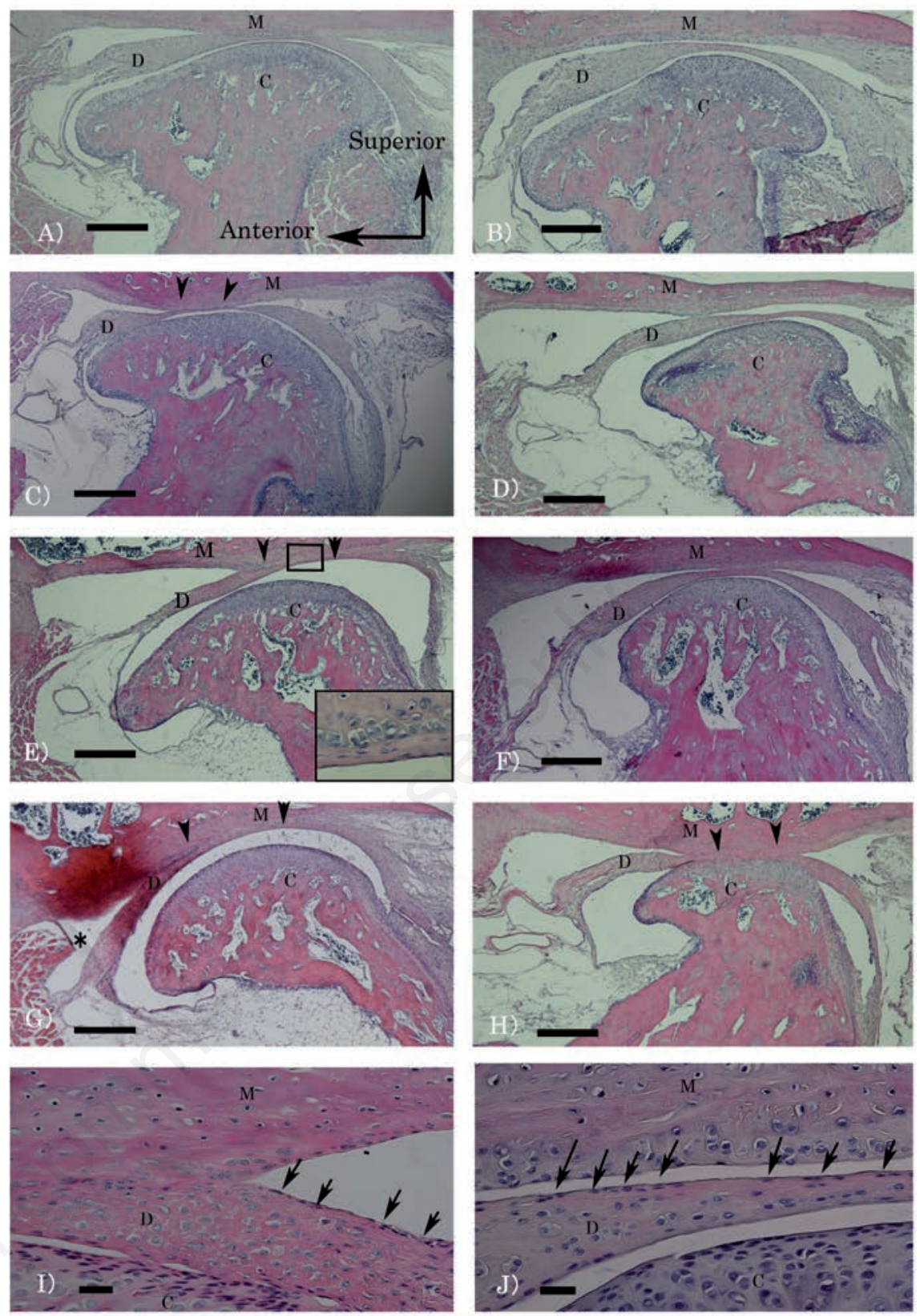

Figure 1. Hematoxylin-eosin staining of the TMJ of WT and TNCKO mice. Samples were collected after 1, 7, 12, and 20 days of excessive mouth opening. A), C), E), G), I) from WT mice. B), D), F), H), J) from TNCKO mice. At day 1, in both TNCKO and WT mice, no growth in the synovial membrane of the upper and lower cavities is observed $(\mathrm{A}, \mathrm{B})$. At day 7 , fibrous adhesion could be seen in the upper cavity in WT mice (C), but not in TNCKO mice (D). At day 12, widespread fibrous adhesion formation is observed in the upper cavity in WT mice (E) and chondrocyte clustering was observed in the mandibular fossa (insert), while the disc of TNCKO mice remained slightly connected to the mandibular fossa (F). At day 20, the upper and anterior portions of the cavity were reduced in WT mice (G), but were unchanged in TNCKO mice $(\mathrm{H})$. Fibroblasts on the articular disc surface disappeared in the area of fibrous adhesion (I). In control, continuous fibroblasts were observed on the articular disc surface $(\mathrm{J})$. Arrows indicate fibroblasts on the disc surface. Arrow heads indicate fibrous adhesion area. M: Mandibular fossa. Asterisk: reduced upper cavity. D, disc; C, condyle. Scale bars: A-H) $200 \mu \mathrm{m}$; I,J) $500 \mu \mathrm{m}$. 
embedded in paraffin. Subsequently, 4- $\mu$ mthick sections were stained with hematoxylineosin (HE). Histological photomicrographs were taken using a digital camera on a Nikon ECLIPSE E600 microscope equipped with a DSL2 controller (Nikon Co. Ltd., Tokyo, Japan).

\section{Immunohistochemical methods}

Immunohistochemical staining was performed on paraffin sections. Endogenous peroxidase was blocked by immersing the sections in $0.3 \% \mathrm{H}_{2} \mathrm{O}_{2}$ in methanol for $30 \mathrm{~min}$ at room temperature (RT). The specimens were blocked with $3 \%$ skim milk in $0.01 \%$ Tween- 20 PBS for $60 \mathrm{~min}$ at RT and were then incubated overnight with a rat monoclonal primary antibody against TNC (1:100, Santa Cruz Biotechnology, Dallas, TX, USA), a rabbit polyclonal primary antibody against FN (1:200, Thermo Scientific, West Palm Beach, FL, USA) and a rabbit polyclonal primary antibody against $\alpha$-SMA (1:200, GeneTex, Inc., Irvine, CA, USA). After washing with Tween-20-PBS, the specimens were treated with the appropriate secondary antibodies (polyclonal rabbit anti-rat immunoglobulins/HRP or polyclonal goat anti-rabbit immunoglobulins/HRP; Dako, Tokyo, Japan) for $60 \mathrm{~min}$ at RT. Color was developed using the Liquid DAB+Substrate Chromogen System (Dako) followed by counterstaining with Mayer's haematoxylin (Muto Pure Chemicals, Tokyo, Japan).

\section{Western blot analysis}

After an overdose of sodium pentobarbital, the right and left TMJ discs were carefully removed using a surgical microscope and washed with PBS. The samples were homogenized in Laemmli sample buffer (0.5 M Tris$\mathrm{HCl}, \mathrm{pH} 6.8,10 \%$ SDS, $0.5 \%$ glycerol), centrifuged at $2000 \mathrm{~g}$ and the supernatants were collected. The samples (10 $\mu \mathrm{g}$ of protein) were electrophoresed on sodium dodecyl sulfate polyacrylamide (10\%) slab gels and transferred onto nitrocellulose membranes (Invitrogen, Carlsbad, CA, USA). The membranes were incubated in $0.01 \%$ Tween-20-PBS containing $3 \%$ skim milk for $1 \mathrm{~h}$ at RT. Next, membranes were incubated with rat monoclonal antibody (1:400) against TNC (Abcam, Cambrige, MA, USA), goat polyclonal antibody (1:100) against FN (Santa Cruz Biotechnology) and rabbit polyclonal antibody (1:100) against $\alpha$-SMA (GeneTex, Inc.) overnight at $4^{\circ} \mathrm{C}$. The membranes were washed three times with Tween20-PBS, and incubated with horseradish peroxidase-conjugated anti-rat IgG (1:100) and anti-goat IgG (1:100) (Dako). The proteins were visualized with ECL prime western blotting detection reagent (GE Healthcare, Stockholm, Sweden) following manufacturer's protocol.

\section{Results \\ Histochemical analysis}

The mouse TMJ is composed of the mandibular fossa, condyle and intervening articular disc, which divide the articular cavity into the upper and lower cavities. The disc of the mouse TMJ is a biconcave structure, composed of an intermediate zone and two bands and attachments. In the intermediate zone, a few spindle-shaped cells were scattered on the disc surface. The surface of the mandibular fossa was covered with a thin fibrous layer, and chondrocytes of the cartilage layer were observed under the fibrous layer. Also, the surface of the condyle was covered with cartilage tissue and a fibrous layer. There were no definite differences in the untreated articular structure between WT and TNCKO mice.

On day 1 post-treatment, contact between the disc and the mandibular fossa was observed in WT mice (Figure 1A), but not in TNCKO mice
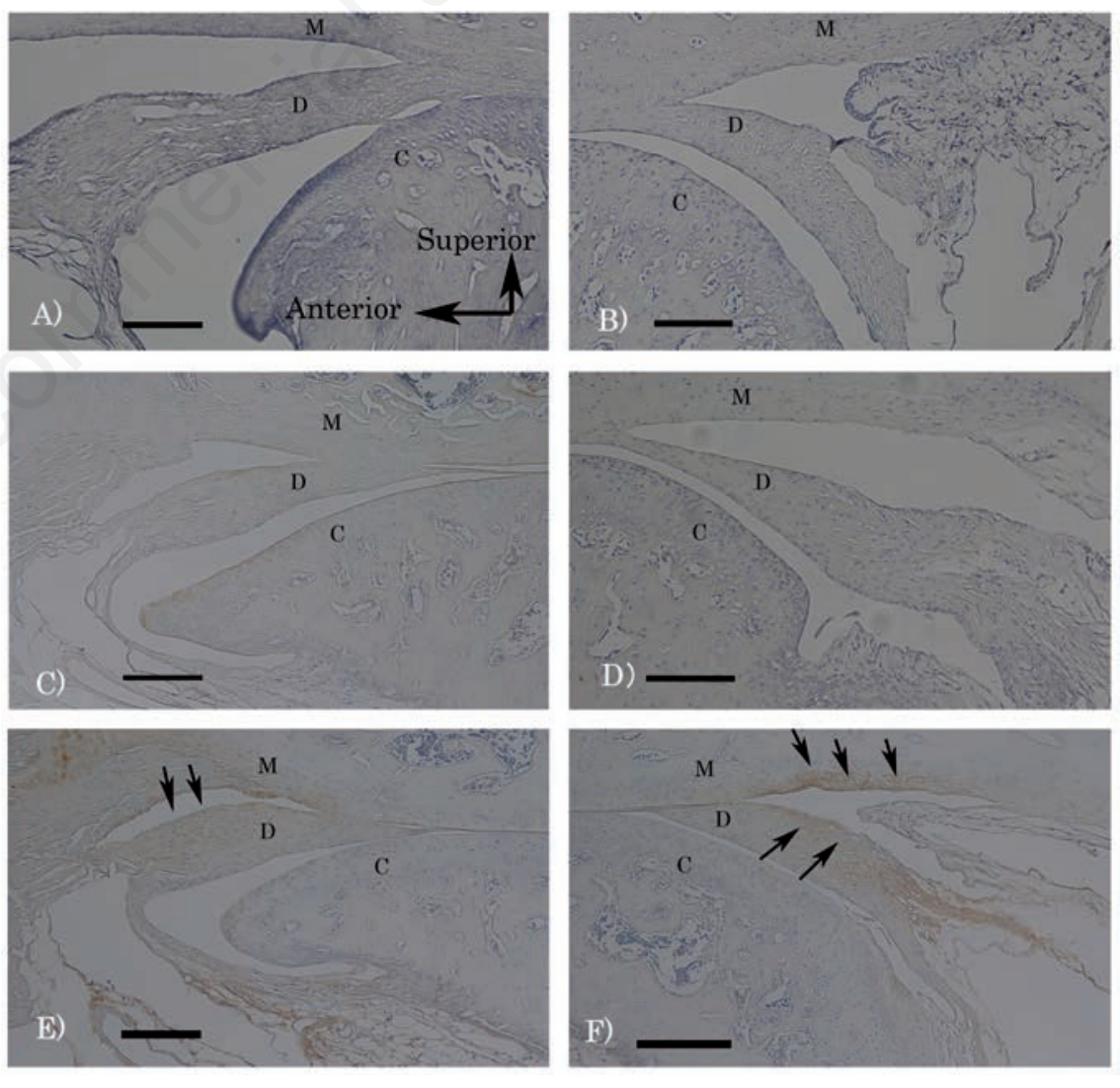

Figure 2. The expression of TNC in the TMJ of WT and TNCKO mice subjected to excessive mouth opening. TNC immunohistochemical staining of the TMJ (sagittal section) of TNCKO mice (A, B), and WT mice (C, D). The expression of TNC on the upper surface of the disc, condyle and mandibular fossa after excessive mouth opening was greater than in control mice $(E, F)$. Arrows indicate expression of TNC. M, mandibular fossa; $D$, disc; C, condyle. Scale bars: $100 \mu \mathrm{m}$. 


\section{Immunohistochemical analysis of TNC, FN and $\alpha$-SMA}

Weak TNC expression was observed in the disc and the fibrous layer covering the condylar and mandibular fossa in control specimens of WT mice (Figure 2 C,D). However, TNC expression increased 1 day after excessive mouth opening. The upper part of the disc showed more intense TNC staining than the lower part in WT mice. TNC expression also increased in the mandibular fossa after excessive mouth opening in comparison with the control specimen of WT mice (Figure 2 E,F). No definite staining was observed in the TMJ of TNCKO mice (Figure 2 A,B). FN positive staining of the TMJ showed differences between WT and TNCKO mice. In the control specimen of WT mice, the surface of the disc and the fibrous layer on the mandibular fossa showed more intense FN staining than that of TNCKO mice (Figure 3 A-D). On day 1 after treatment, the WT sample showed intense FN staining at the disc and the fibrous layer on the mandibular fossa (Figure $3 \mathrm{E}, \mathrm{F}$ ), whereas the TNCKO sample showed weak FN staining (Figure $3 \mathrm{G}, \mathrm{H}$ ). $\alpha$-SMA positive staining of the TMJ showed differences between WT and TNCKO mice. In the control specimen of WT mice, the disc and the fibrous layer on the mandibular fossa showed moderate $\alpha$-SMA staining than that of TNCKO mice (Figure $4 \mathrm{~A}$ D). On day 12 after treatment, the WT sample showed moderate $\alpha$-SMA staining at the disc and the fibrous layer on the mandibular fossa (Figure $4 \mathrm{E}, \mathrm{F}$ ), whereas the TNCKO sample showed weak FN staining (Figure $4 \mathrm{G}, \mathrm{H}$ ).

\section{Western blot analysis of TNC, FN and $\alpha-S M A$}

The western blot analysis of disc tissue revealed a single $230 \mathrm{kDa}$ band for TNC expressed after treatment. TNC expression was very low in the control, but increased markedly after treatment from day 1 to day 20 . Consistent with expectations, TNC was not detected in the TNCKO samples (Figure 5). The western blot results showed a single band of FN corresponding to a molecular weight of $220 \mathrm{kDa}$ in WT and TNCKO samples (Figure 6). The expression of FN was very low in control, but increased markedly after treatment. The relative intensities of the $\mathrm{FN}$ bands were stronger in WT samples than in TNCKO samples. Additionally, the western blot results showed a single band of $\alpha$-SMA corresponding to a molecular weight of $42 \mathrm{kDa}$ in WT and TNCKO samples (Figure 7). The expression of $\alpha$-SMA was moderate in control, but increased evidently at 12 days after treatment in WT sample. The relative intensities of the $\alpha$-SMA bands were stronger in WT samples than in TNCKO samples at day 12 after treatment.

\section{Discussion}

The role of TNC in fibrous adhesion formation in the TMJ is not yet known. Trying to clarify this, we compared the TMJ wound healing process of WT and TNCKO mice after excessive mouth opening. In this study, we demonstrated that TNC promotes fibrous adhesion formation in the TMJ disc with excessive mouth opening. Furthermore, TNC appears to enhance the expression of FN and $\alpha$-SMA, and a lack of TNC may reduce fibrous adhesion formation in the TMJ.
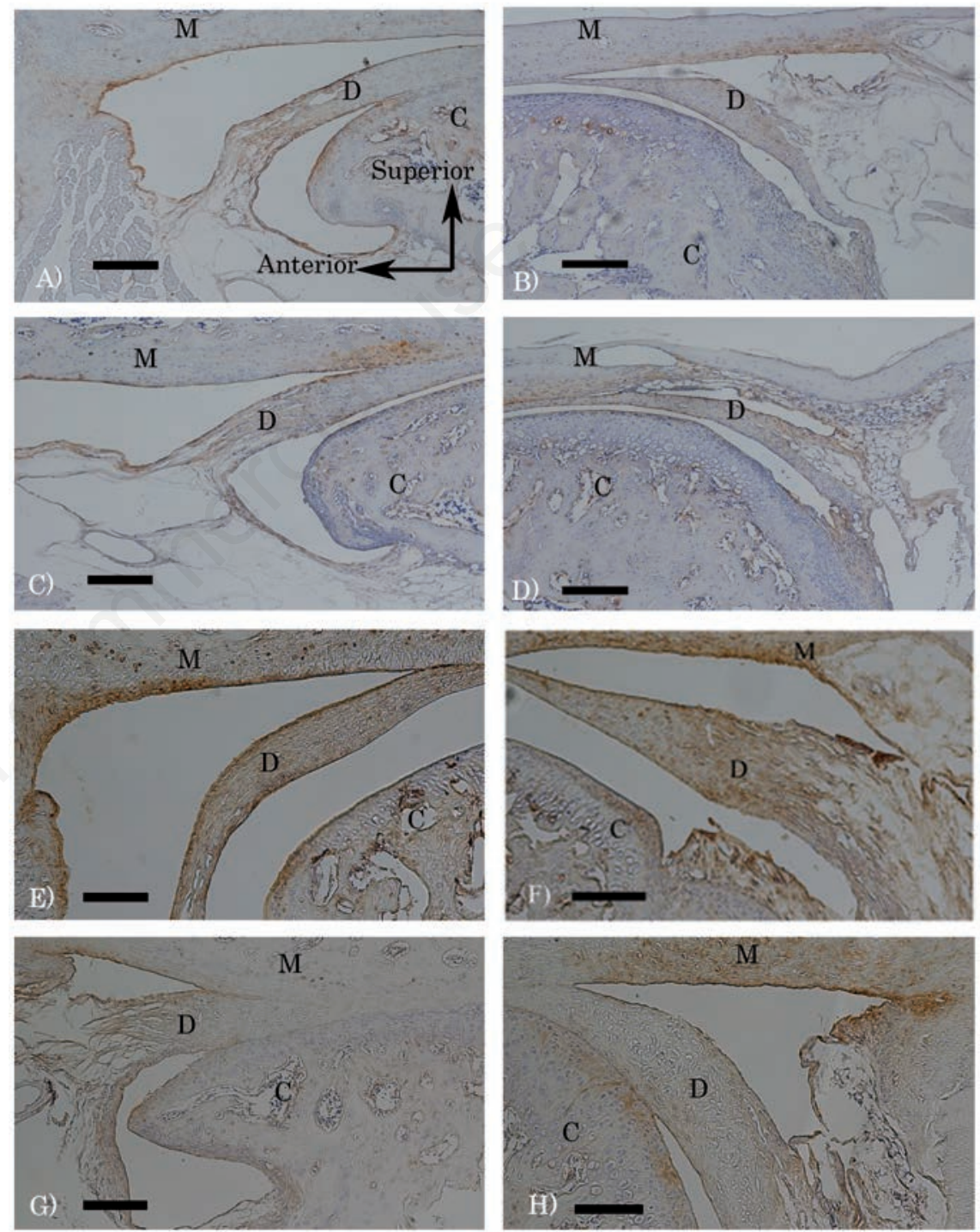

Figure 3. The expression of FN in the TMJ of WT and TNCKO mice subjected to excessive mouth opening. FN expression in the control TMJ of WT (A, B) and TNCKO mice (C, D). The surface of the disc and fibrous membrane of the mandibular fossa showed more intense FN staining in WT mice than in TNCKO mice. The TMJ sections at day 1 showed intense FN staining at the disc and the fibrous membrane on the mandibular fossa (E, F), whereas that of TNCKO mice showed a faint amount of FN staining $(G, H) . M$, mandibular fossa; $\mathrm{D}$, disc; C, condyle. Scale bars: $100 \mu \mathrm{m}$. 
mation of scar tissue on the existing fibrous capsular wall. In a previous report on the developmental and histological characteristics of synovitis, adhesions were induced in the synovium in the anterior portion of the cavity in the rat TMJ by excessive mouth opening. ${ }^{25}$ However, detection of synovitis was based on increased synovial membrane and fibrin deposition; the existence of hematomas was not observed during our experimental period. In the current mouse study, the formation and progressive maturation of adhesions was induced between the mandibular fossa and the disc, instead of in the synovium, and was directly related to the duration of excessive mouth opening. These differences may depend on the extent of damage, length of treatment or anatomy of the animal species. Fibrous adhesion formation was initiated 7 days after the last treatment in the upper cavity and continued for 20 days in WT mice. The possibility exists that excessive mouth opening causes microtrauma due to excessive pressure on the disc by the hyper movement of the mandibular condyle and mandibular fossa. This microtrauma may cause the fibrin related adhesion, eventually developing into a fibrous adhesion. Fibroblasts, which exist in the fibrous layer of the mandibular fossa and disc, are thought to form collagen fibers and facilitate fibrous adhesion formation. Twelve days after the last treatment in WT mice, the extent of adhesion formation was increased with the clustering of chondrocytes in the cartilage layer of mandibular fossa (Figure 1E; see insert). Generally, chondrocyte clustering is a characteristic feature of the early stage repair process in the cartilage layer in osteoarthritis, ${ }^{33,34}$ and is a response that produces extracellular matrix components in the presence of mechanical stress on the chondrocytes. Throughout the experimental period, fibrous adhesions were not observed in the lower joint cavity. As this adhesion pattern resembles that of humans, excessive mouth opening is thought to cause mechanical stress in the upper part of the articular disc. On the other hand, initiation of fibrous adhesion formation in the disc of TNCKO mice was delayed in comparison to WT mice. Also, adhesion length did not increase throughout the experimental period, as was observed in WT mice. A reduction of the upper cavity area was not observed in TNCKO mice over the study period. In conclusion, while fibrous adhesions developed in WT mice, the fibrotic changes in TNCKO mice were clearly less extensive. These results indicate that TNC promotes fibrous adhesion formation in discs upon excessive mouth opening.

It is well known that FN is a major adhesive glycoprotein involved in wound healing and its up-regulation is related to histological changes. Therefore, we compared the expression and changes in protein levels of TNC and FN in mice after induced hypermobility using immunohistochemical and western blotting analyses. Western blotting analysis showed dramatic increases in TNC and FN protein levels after excessive mouth opening in WT mice, which were maintained at a fixed level throughout the experimental period. Although the intensity of the FN band in TNCKO mice was extremely low in comparison to WT mice, mild up-regulation of FN protein was observed in TNCKO mice. TNC was expressed in the TMJ disc, mandibular fossa
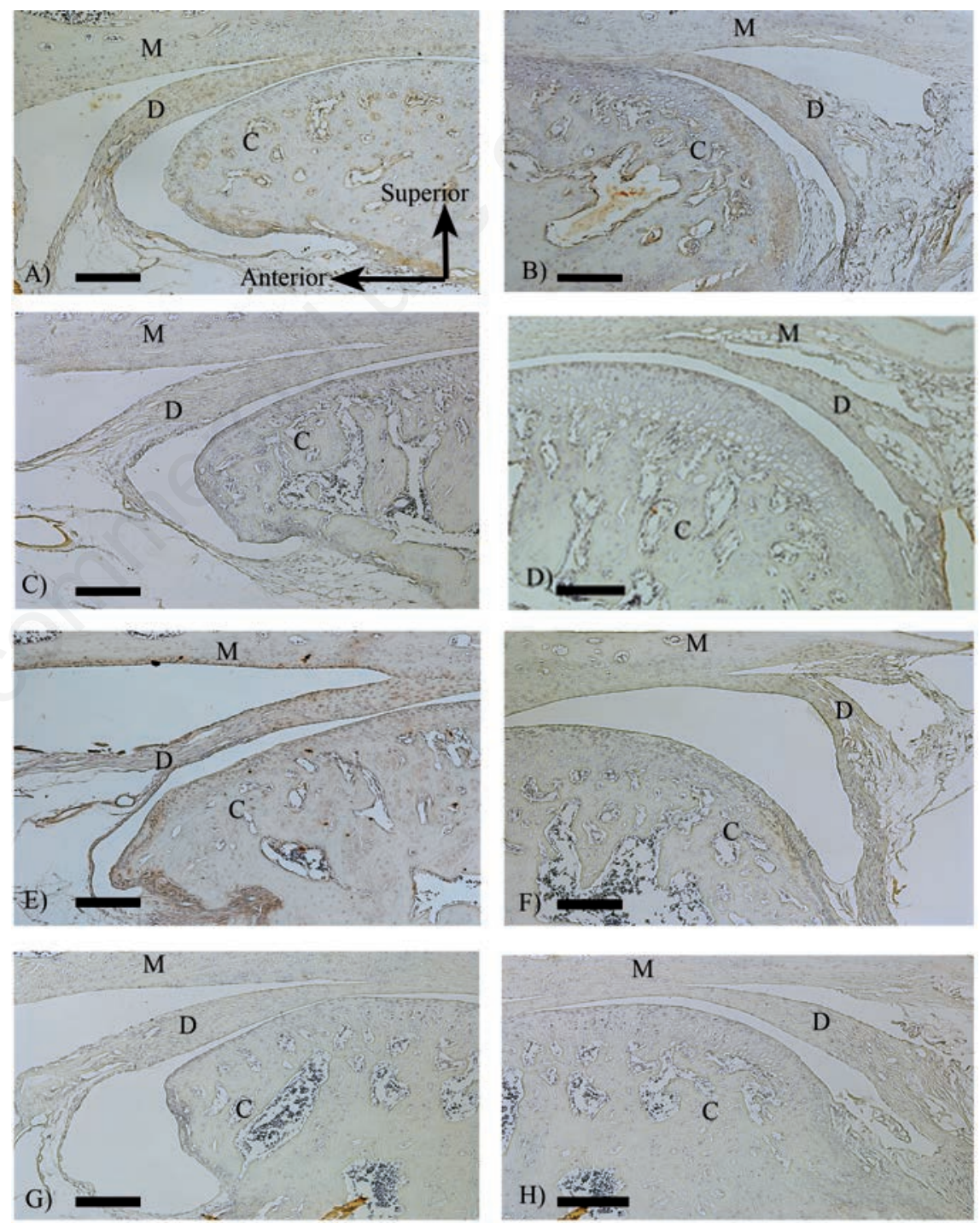

Figure 4. The expression of $\alpha$-SMA in the TMJ of WT and TNCKO mice subjected to excessive mouth opening. $\alpha$-SMA expression in the control TMJ of WT (A, B) and TNCKO mice (C, D). The TMJ sections at day 12 showed intense moderate $\alpha$-SMA staining at the disc and the fibrous membrane on the mandibular fossa (E, F), whereas that of TNCKO mice showed a faint amount of $\alpha$-SMA staining $(G, H)$. M, mandibular fossa; D, disc; C, condyle. Scale bars: $100 \mu \mathrm{m}$. 


\section{Control Day 1 Day 7 Day12 Day20}

TNC

WT

ßactin

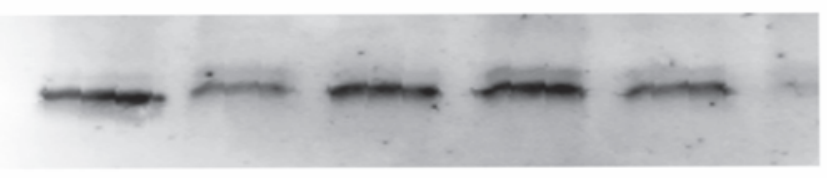

TNC

\section{TNCKO}

B-actin
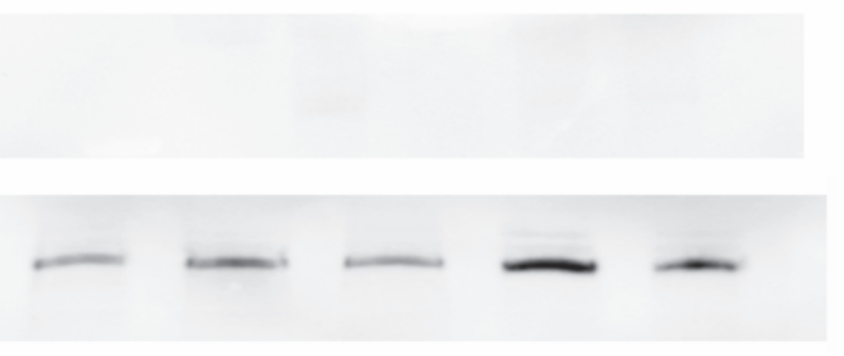

Figure 5. Chronological alterations in TNC expression in WT and TNCKO mice after excessive mouth opening. Western blot analysis showed a TNC band corresponding to 230 $\mathrm{kDa}$ in control, and day $1,7,12$, and 20 samples from WT mice. However, a band corresponding to TNC was not detected in TNCKO samples. TNC expression increased after excessive mouth opening in WT samples.

\section{Control Day1 Day7 Day12 Day20}

FN

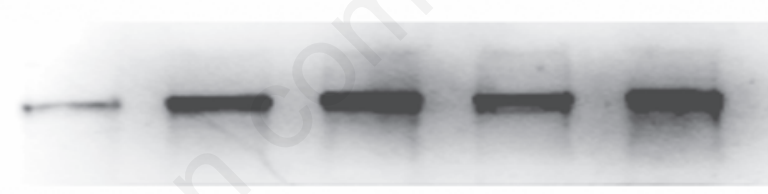

WT

B-actin

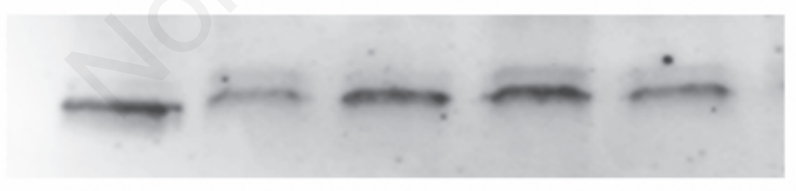

FN

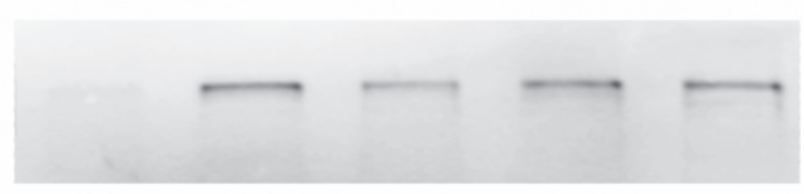

TNCKO

B-actin

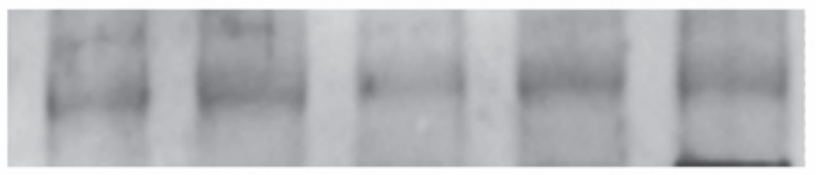

Figure 6. Chronological alterations in FN expression in WT and TNCKO mice after excessive mouth opening. Western blot analysis showed a FN band corresponding to $220 \mathrm{kDa}$ in control, and day 1,7, 12 and 20 samples from WT mice and TNCKO mice. Band intensity was stronger in WT samples than in the TNCKO sample at identical time points.
TNC expression. The expression pattern of FN in the superficial layer of the mandibular fossa and the disc was consistent with adhesion formation after treatment in both WT and TNCKO mice. Other studies have shown that the increase in tissue TNC levels can be used as a predictive marker for fibrosis. ${ }^{35}$ TNCKO mice exhibit thinner stroma during corneal suture wounds with reduced FN depositon. ${ }^{36}$ Similarly, lower levels of FN were detected in the granulation tissue during healing of dermal excisional wounds in TNCKO mice, ${ }^{37}$ and only small and immature granulation tissue was observed during experimental kidney injury. ${ }^{29}$ One reason for the delayed and decreased adhesion formation in the TMJ in TNCKO mice may depend on decreased FN protein levels.

Fibroblastic cells acquire contractile features during the healing of a wound, thus modulating into myofibroblasts which synthesize extracellular matrix components such as collagen type I and type III, and during normal wound healing disappear by apotosis when epithelialization occurs. The transition from fibroblasts to myofibloblasts is influenced by mechanical stress. ${ }^{38}$ Myofibroblasts are considered the main effector cell in joint fibrosis. One articular process (adhesive capsulitis of the shoulder) and two non-articular process (Dupuytren's contracture of the hand and hypertrophic scarring) have been shown to lead to nontraumatic contractures. ${ }^{39-41}$ In both WT and TNCKO mice, the expression level of protein of $\alpha$-SMA became maximam at 12 days after excessive mouth opening. The time $\alpha$-SMA strongly expressed was in agreement with the time which the fibrous adhesion range expands. Therefore, $\alpha$-SMA may be a factor related to formation of fibrous adhesion of mice disc. The intensity of the $\alpha$-SMA expression in TNCKO mice was extremely low in comparison to WT mice. We consider the delayed and decreased adhesion formation in the TMJ in TNCKO mice may depend on decreased $\alpha$-SMA protein levels. The expression of lumican, which is an extracellular matrix proteoglycan, increased in an inflammatory model using an articular disc cell line. ${ }^{42}$ Therefore, differences may exist in the expression of lumican in WT and TNCKO mice. Thus, it is thought that several genes participate in adhesion formation in TMJ. Further in vitro studies are needed to elucidate the mechanism by which TNC affects the expression of other extracellular matrix proteins. 


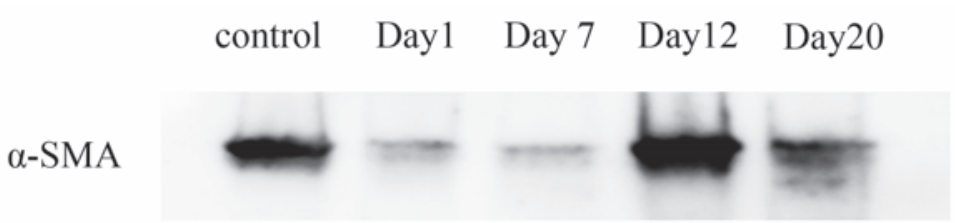

WT $\beta$-actin

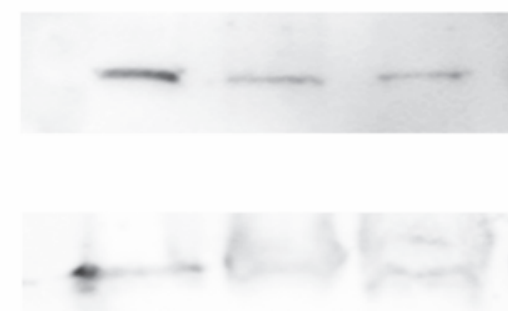
$\alpha$-SMA

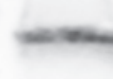

TNCKO

$$
\beta \text {-actin }
$$
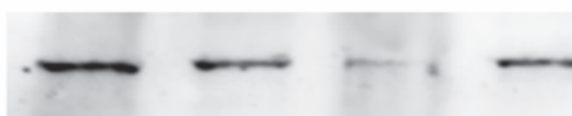

Figure 7. Chronological alterations in $\alpha$-SMA expression in WT and TNCKO mice after excessive mouth opening. Western blot analysis showed a $\alpha$-SMA band corresponding to 42 $\mathrm{kDa}$ in control, and day 1, 7, 12 and 20 samples from WT mice and TNCKO mice. Band intensity was stronger in WT samples than in the TNCKO sample at day 12 after treatment.

\section{References}

1. Ronald M, Kaminishi RM, Davis CL. Temporomandibular joint arthroscopic observation of superior space adhesions. Oral Maxillofac Surg Clin North Am.1989;1:103-9.

2. Kurita K, Bronstein SL, Westesson PL, Sternby NH. Arthroscopic diagnosis of perforation and adhesions of the temporomandibular joint: Correlation with postmortem morphology. Oral Surg Oral Med Oral Pathol 1989;68:130-4.

3. Murakami K, Segami N, Moriya Y, lizuka T. Correlation between pain and dysfunction and intra-articular adhesions in patients with internal derangement of the temporomandibular joint. J Oral Maxillofac Surg 1992;50:705-8.

4. Smartt JM Jr, Low DW, Bartlett SP. The pediatric mandible: I. A primer on growth and development. Plast Reconstr Surg 2005;116:14-23e.

5. Arnett GW, Milam SB, Gottesman L. Progressive mandibular retrusion--idiopathic condylar resorption. Part I. Am J Orthod Dentofacial Orthop 1996;110:8-15.

6. Stegenga B, DeBont LGM, Boering G. Osteoathrosis as the cause of craniomandibular pain and dysfunction: a unifying concept. J Oral Maxillofac Surg 1989;47:249-56.

7. Chiquet-Ehrismann R. Tenascins. Int $\mathbf{J}$ Biochem Cell Biol 2004;36:986-90.

8. Tucker RP, Chiquet-Ehrismann R. The reg- ulation of tenascin expression by tissue microenvironments. Biochim Biophys Acta 2009;1793:888-92.

9. Midwood KS, Orend G. The role of tenascin- $\mathrm{C}$ in tissue injury and tumorigenesis. J Cell Commun Signal 2009;3:287-310.

10. Tojyo I, Yamaguti A, Nitta T, Yoshida H, Fujita S, Yoshida T. Effect of hypoxia and interleukin-1 $\beta$ on expression of tenascin$\mathrm{C}$ in temporomandibular joint. Oral Diseases 2008;14:45-50.

11. Sato I, Uneno R, Miwa Y, Sunohara M. Distribution of tenascin- $C$ and tenascin- $X$, apoptotic and proliferating cells in postnatal soft-diet rat temporomandibular joint. Ann Anat 2006;188:127-36.

12. Milam SB, Klebe RJ, Triplett RG, Herbert D. Characterization of the extracellular matrix of the primate temporomandibular joint. $\mathrm{J}$ Oral Maxillofac Surg. 1991;49:381-91.

13. Leonardi R, Michelotti A, Farella M, Caltabiano R, Lanzafame S. Fibronectin upregulation in human temporomandibular joint discs with internal derangement. J Craniofac Surg. 2004;15:678-83.

14. Milam SB, Haskin C, Zardeneta G, Chen D, Maqnuson VL, Klebe RJ, et al. Cell adhesion proteins in oral biology. Crit Rev Oral Biol Med. 1991;2:451-91.

15. Dijkgraaf LC, Zardeneta G, Cordewener FW, Liem RS, Schmitz JP, de Bont LG, et al. Crosslinking of fibrinogen and fibronectin by free radicals: a possible initial step in adhesion formation in osteoarthritis of the temporomandibular joint. J Oral Maxillofac Surg 2003;61:101-11.
16. Serini G, Gabbiani G. Mechanisms of myofibroblast activity and phenotypic modulation. Exp Cell Res 1999; 250:273283.

17. Majno G, Gabbiani G, Hrschel BJ, Ryan GB, Statkov PR. Contraction of granulation tissue in vitro: similarity to smooth muscle. Science 1971;173:548-550.

18. Gabbiani G, Majino G. Dupuytren's contracture: fibroblast contraction? An ultrastructural study. Am J Pathol 1972;66:131-46.

19. Jester JV, Petroll WM, Cavanagh HD. Corneal stromal wound healing in refractive surgery: the role of myofibroblasts. Prog Retin Eye Res 1999;18:311-56.

20. Serini G, Gabbiani G. Mechanisms of myofibroblast activity and phenotypic modulation. Exp Cell Res 1999;250:273-83.

21. Tomasek JJ, Gabbiani G, Hinz B, Chaponnier C, Brown RA. Myofibroblasts and mechanoregulation of connective tissue remodeling. Mol Cell Biol 2002;3:349-63.

22. Teramoto M, Kaneko S, Shibata S, Yanagishita M, Soma K. Effect of compressive forces on extracellular matrix in rat mandibular condylar cartilage. J Bone Miner Metab 2003;21:276-86.

23. Honzawa 0 . [Effects of bite raising on fine structure of condylar cartilage in rats]. [Article in Japanese]. Nihon Kyousei Shika Gakkai Zasshi1989;48:29-47.

24. Endo Y, Mizutani H, Yasue K, Ueda M. Influence of food consistency and dental extractions on the rat mandibular condyle: a morphological, histological and immunohistochemical study. J Craniomaxillofac Surg 1998;26;185-90.

25. Muto T, Kawakami J, Kanazawa M, Kaku T, Yajima T. Development and histologic characteristics of synovitis induced by trauma in the rat temporomandibular joint. Int $\mathrm{J}$ Oral Maxillofac Surg 1998;27:470-5.

26. Siga T, Ishibashi K, Toyoda N, Nogami N, Ishii $\mathrm{H}$, Ohotake $\mathrm{M}$, et al. A production of experimental fibrous adhesions of the temporomandibular joint of the rabbit and its histological study. J Jpn Soc TMJ 2002;14:210-6.

27. Saga Y, Yagi T, Ikawa Y, Sakakura T, Aizawa S. Mice develop normally without tenascin. Genes Dev 1992;6:1821-31.

28. Cifuentes-Diaz C, Velasco E, Meunier FA, Goudon D, Belkadi L, Faille L, et al. The peripheral nerve and the neuromuscular junction are affected in the tenascin-Cdeficient mouse. Cell Mol Biol 1998;44: 357-79.

29. Nakao N, Hiraiwa N, Yoshiki A, Ike F, Kusakabe M. Tenascin-C promotes healing of Habu-snake venom-induced glomerulonephritis: studies in knockout congenic mice and in culture. Am J Pathol 
1998;152:1237-45.

30. Koyama Y, Kusubata M, Yoshiki A, Hiraiwa $\mathrm{N}$, Ohashi T, Irie S, et al. Effect of tenascin$\mathrm{C}$ deficiency on chemically induced dermatitis in the mouse. J Invest Dermatol 1998;111:930-5.

31. El-Karef A, Yoshida T, Gabazza EC, Nishioka T, Inada H, Sakakura T, et al. Deficiency of tenascin-C attenuates liver fibrosis in immune-mediated chronic hepatitis in mice. J Pathol 2007;211:86-94.

32. Imanaka-Yoshida K, Matsumoto K, Hara M, Sakakura T, Yoshida T. The dynamic expression of tenascin- $C$ and tenascin- $X$ during early heart development in the mouse. Differentiation 2003;71:291-8.

33. Fujii K, Kuboki Y, Sasaki S. Aging of human bone and articular cartilage collagen: changes in the reducible cross-links and their precursors. Gerontology 1976;22:363-70.

34. Mankin HJ. The response of articular car- tilage to mechanical injury. J Bone Joint Surg Am 1982;64:460-6.

35. Leiber CS, Weiss DG, Paronetto F. Value of fibrosis markers for staging liver fibrosis in patients with precirrhotic alcoholic liver disease. Alcohol Clin Exp Res 2008;32: 1031-9.

36. Mastuda A, Yoshiki A, Tagawa Y, Matuda H, Kusakabe M. Corneal wound healing in tenascin knockout mouse. Invest Ophthalmol Vis Sci 1999;40:1071-80.

37. Forsberg E, Hirsch E, Fröhlich L, Meyer M, Ekblom P, Aszodi A, et al. Skin wounds and severed nerves heal normally in mice lacking tenascin-C. Proc Natl Acad Sci USA 1996;93:6594-9.

38. Gabbiani G. The myofibroblast in wound healing and fibrocontractive disease. J Pathol 2003;200:500-3.

39. Berndt A, Kosmehl H, Katenkamp D, Tauchmann V. Appearance of the myofibroblastic phenotype in Dupuytren's dis- ease is associated with a fibronectin, laminin, collagen type IV and tenascin extracellur matrix. Pathobiology 1994; 62:55-8.

40. Rodeo SA, Hannafin JA, Tom J, Warren RF, Wickiewicz TL. Immunolocalization of cytokines and their receptors in adhesive capsulitis of the shoulder. J Orthop Res 1997;15:427-36.

41. Nedelec B, Shankowsky H, Scott PG, Ghahary A, Tredget EE. Myofiboblasts and apoptosis in human hypertrophic scars: the effect of interferon-alpha 2b. Surgery 2001;130:798-808.

42. Kiga N, Tojyo I, Matsumoto T, Hiraishi Y, Shinohara Y, Makino S, et al. Expression of lumican and fibromodulin following interleukin-1 beta stimulation of disc cells of the human temporomandibular joint. Eur J Histochem 2011;55:e11. 